\title{
Determination of temperature and density of the stars using the new intensity formula
}

\author{
Bo Thelin
}

\begin{abstract}
In this paper a linear relationship in stellar optical spectra has been found by using a spectroscopical method used on optical light sources where it is possible to organize atomic data in a linear structure. This method is based on a new intensity formula in optical emission spectroscopy (OES). Like the HR-diagram, it seems to be possible to organize the luminosity of stars from different spectral classes. From that organization it is possible to determine the temperature and density of stars using the new intensity formula. These temperature -and density values agree well with literature values. It is also possible to determine the mean electron temperature of the optical layers (photospheres) of the stars as it is for atoms in the laboratory plasmas. The mean value of the ionization energies of the different elements of the stars has shown to be very significant for each star. This paper also shows that the hydrogen Balmer absorption lines follow the new intensity formula.

Keywords: stellar optical spectra, linear relationship, temperature measurements and density measurements.
\end{abstract}

\section{Introduction}

The author and the collegue Dr. Sten Yngström have earlier presented a new formula for the intensity of spectral lines in optical emission spectroscopy (OES) in many previous papers and conferences. According to a new theory in Ref 1 the intensity $\mathrm{I}(\mathrm{h} v)$ is given by equation 1

$$
I(\text { hv })=C \lambda^{-2} \exp (-\mathrm{J} / \mathrm{kT}) /((\exp (\mathrm{hv} / \mathrm{kT})-\mathbf{1})
$$

where $v$ is the frequency of the $\lambda$ is the wavelength of atomic spectral line, $J$ the ionization energy of the atom, and $\mathrm{C}$ is a product of factors about sample properties (number densities of atoms and electrons) and the transition probability of the atom.

In earlier papers by us about this formula in $\operatorname{Ref}(2)$ and $\operatorname{Ref}(3)$, we studied absolute intensities. The intensities came from arc measurements and are tabled in Ref (4), which we have used in our studies. In these studies the new intensity formula was used in the development of this method of analysis. In this method $\ln \left(\mathrm{I} \lambda^{2}\right)$ was plotted versus $\mathrm{h} v(1+\theta / \mathrm{h} v \ln (1-\exp (-\mathrm{h} v / \theta))) \mathrm{eV}$ for 17 elements.

Each intensity value is the mean value of many individual values. By forming the maximum between the difference between $\ln I \lambda^{2}$ and $\ln \lambda^{2}$ the following formula will be the basic equation in this method of analysis.

$$
\ln \left(I_{\max } \lambda^{2}{ }_{\max }\right)=\text { const. }-1.6 \mathrm{~J} / \mathrm{h} v_{\max } \quad \text { ( 2 ) }
$$

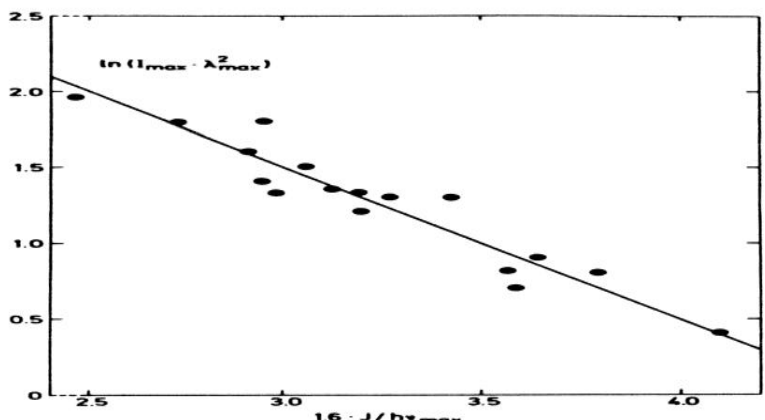

Fig $1 \ln \left(I_{\max } \lambda^{2}{ }_{\max }\right)$ plotted versus (1.6 J ) / hv $\max$ for seventeen elements from the NBS tables in Ref 4.

This graph can be seen in Fig 1, where $\ln \left(\mathrm{I}_{\max } \lambda^{2}{ }_{\max }\right)$ has been plotted versus $1.6 \mathrm{~J} / \mathrm{h} v_{\max }=\mathrm{J} / \theta$ for 17 elements, where $\theta=\mathrm{k} \mathrm{T}_{\mathrm{e}}$ (electron temperature). $\mathrm{J}$ denotes table value of ionization energy. This graph forms a good linear relationship, where $h v_{\max }=1.6 \theta$. This means that this graph is a strong support of the new intensity formula, based on the new theory. It is also possible to measure the internal electron temperature for different elements. It has now shown to be possible to obtain similar linear relationships when using intensity 
data of stellar optical spectra. In Table 1 is shown the electron temperature and ionization energies values from 17 elements with this method.

A very strong support of this new intensity formula has recently been published in two open access summary papers Ref 5 and Ref 6 , where different methods from the literature have been used which support the new intensity formula.

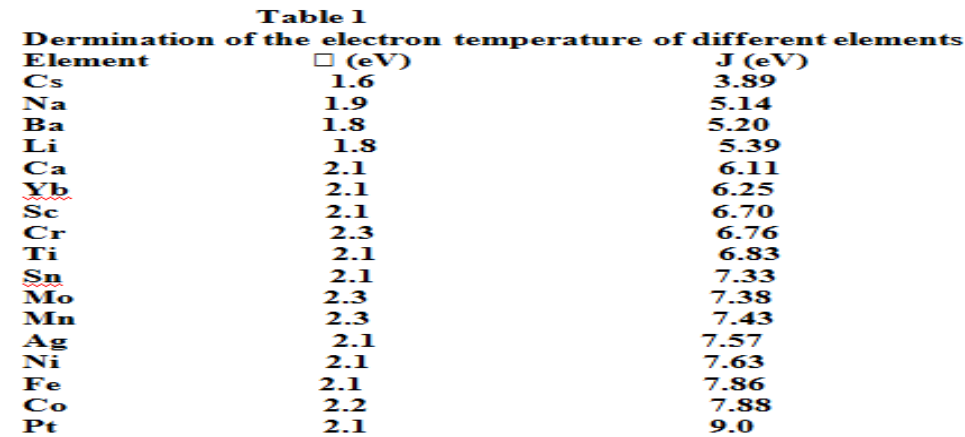

The electron temperature values from Table 1 have a mean value of about $2 \mathrm{eV}$, which fits well literature values in $\operatorname{Ref} 7$.

\section{Stellar spectra}

These stellar optical spectra extend over the spectral classes $\mathrm{O}-\mathrm{M}$ and the photometrically well-calibrated luminosity measurements from star to star, and come from Ref 8 . Good temperature and luminosity coverage have been achieved. The data were digitalized from the main sequence classed O5 - F0 and F6 - K5 displayed in term of relative flux as a function of wavelength. The parameters that have been measured in this investigation are maximum luminosity $\mathrm{L}_{\max }$ (Rel.fluxmax) of the Planck curve. In this maximum the wavelength $\lambda_{\max }$ and the maximum frequency $v_{\max }$ were also measured.

Then $\ln \left(\mathrm{L}_{\max } \lambda_{\max }{ }^{2}\right)$ values were plotted versus $\left(1.6 \mathrm{~J}_{\text {meanvalue }} / \mathrm{h} v_{\max }\right)$ where $\mathrm{J}_{\text {meanvalue }}$ is the mean value of the ionization energies of the elements of the stars measured. To obtain a similar linear relationship for the stellar data as Fig 1 from the spectroscopical method from Refs 2 and 3, the following luminosity data from Ref 8 and data from Table 2 were used and plotted according to equation 3

$$
\ln \left(L_{\max } \lambda_{\max }{ }^{2}\right)=\text { const }-\left(1.6 J_{\text {meanvalue }} / \mathbf{h} v_{\max }\right)
$$

which is similar to equation 2 for atoms.

To obtain the values of Table 2 it is necessary to use a two step procedure. In the first step it is necessary to define the graph by calculating the $\mathrm{J}_{\text {meanvalue }}$ of the $\mathrm{G} 2$-star. The $\mathrm{J}_{\text {meanvalue }}$ can be expressed in the following way:

$$
\mathbf{J}_{\text {meanvalue }}=\sum \mathbf{c}_{\mathbf{n}} \mathbf{J}_{\mathbf{n}}
$$

where $c_{n}$ is the normalized content of an element of a star. It is plausible to consider the content values of G2stars rather to be similar to the content values of the sun. Therefore the $c_{n}$-values of the sun have been used here. $J_{n}$ is here the ionization energy of an element of a star. This $J_{\text {meanvalue }}$ has been calculated for the sun $(G 2$ star), which gave $J_{\text {meanvalue }}=16.2 \mathrm{eV}$ according to the linear graph in Fig 2 . This value is $16.2 \mathrm{eV}$, too, for the sun when using equation 4 together with established chemical composition values of the sun. This means that we now have one point determined in Fig 2.

A more profound description of this method of creating Fig 2 and Table 2 is described in Ref 9

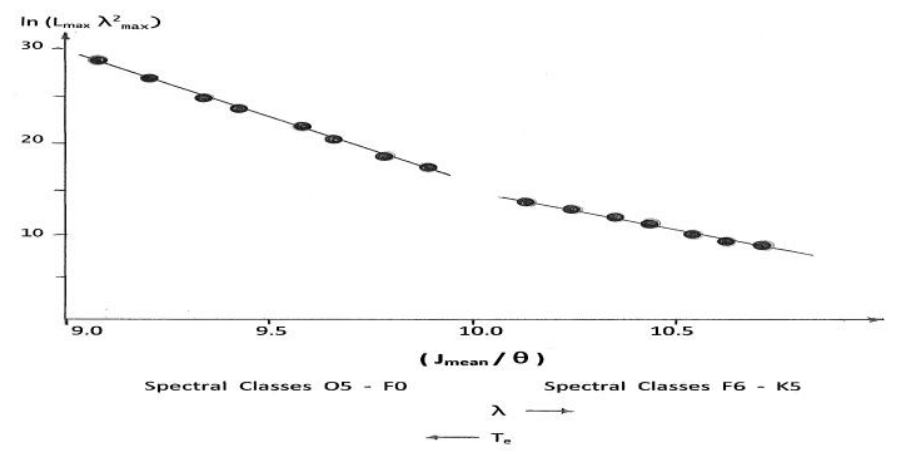




\section{Fig $2 \ln \left(L_{\max } \lambda^{2}{ }_{\max }\right)$ plotted versus (1.6 $\left.J_{\text {meanvalue }}\right) / \mathbf{h} v_{\max }$ for different stars from spectral classes $\mathrm{O}-\mathrm{M}$ from ref $(9)$}

The data in Fig 2 constitute a straight line in the classes O5 - F0 and F6 - K5 in accordance with the usual HRdiagram.

In equation $3 \mathrm{~h} v_{\max }=1.6 \theta$, where $\theta=$ internal electron temperature in $\mathrm{eV}$. This means that the classes $\mathrm{O} 5-\mathrm{F} 0$ have higher temperature than the classes F6 - K5, which is also in accordance with the usual HR-diagram. For example a $\mathrm{G} 2$ star (the sun) has $\theta=1.56 \mathrm{eV}\left(\mathrm{T}_{\mathrm{e}}=18110 \mathrm{~K}\right)$.

Table 2

$\begin{array}{ccc}\text { Determination of the electron temperature of the stars from different spectral classes } \\ \text { Spectral class } & & \\ \text { K5V } & (\mathbf{e V}) & \mathbf{J}_{\text {meanxalue }}(\mathbf{e V}) \\ \text { K4V } & 1.44 & 15.5 \\ \text { G9-K0 } & 1.47 & 15.6 \\ \text { G6-G8 } & 1.50 & 15.8 \\ \text { G1-G2 } & 1.53 & 16.0 \\ \text { F8-F9V } & 1.56 & 16.2 \\ \text { F6-F7V } & 1.63 & 16.7 \\ \text { A9-F0V } & 1.63 & 16.5 \\ \text { A8 } & 1.72 & 16.9 \\ \text { A5-A7 } & 1.75 & 17.1 \\ \text { A1-A3 } & 1.81 & 17.5 \\ \text { B6V } & 1.84 & 17.6 \\ \text { B3-B4V } & 1.88 & 17.8 \\ \text { O7-B0V } & 1.94 & 18.0 \\ \text { O5V } & 1.97 & 18.1 \\ \end{array}$

III. The use of the Balmer lines

It is shown in the in the paper by Ref 8 that the intensity maximum of the continuous-and discrete spectra of stars seem to be the same, where the hydrogen Balmer absorption lines of different stars have been studied. These are the well known Planck curves with steep low wavelength side and a slow high wavelength side. The wavelength of the intensity maximum of continuous-and discrete spectrum seems to be the same. This in agreement with equation 1 and the new theory, where the Planck factor is a part of the new intensity formula. This is clearly seen in Fig 3 from the spectrum of two A-stars. The normalized flux is here proportional to the emissions from the continuous-and discrete spectrum. These curves show very good examples of Planck curves, where continuous-and discrete emissions seem to have the same wavelength maximum.

The wavelengths of the Balmer lines are shown in Table 3 (Ref 10). By using equation 3 and Table 2 intensity ratios have been determined theoretically(from intensity formula) and experimentally by using the data of Ref 8 , from different spectral classes of stars. At the use of these intensity ratios $\mathrm{J}_{\mathrm{H}}=13.595 \mathrm{eV}$ for hydrogen was used. The electron temperatures for different spectral classes have earlier been determined in Tab 1 in Ref 9. A summary of the values from the spectral classes of this paper is shown in Table 4. Nice correlation $(\mathrm{r}=0.98)$ has been achieved between theoretical-and experimental intensity ratios.

This is shown in Fig 4 and is, together with Fig 3 , a strong evidence of the fact that stars follow the new intensity formula, as atoms and ions do. Fig 4 shows very nice correlation between experiment and theory.

Table 3

\begin{tabular}{cc}
\multicolumn{3}{c}{ Balmer lines used } \\
$\mathrm{H}_{\alpha}$ & $6562.80 \AA$ \\
$\mathrm{H}_{\beta}$ & $4861.32 \AA$ \\
$\mathrm{H}_{\gamma}$ & $4340.46 \AA$ \\
$\mathrm{H}_{\delta}$ & $4101.73 \AA$ \\
$\mathrm{H}_{\varepsilon}$ & $3970.07 \AA$
\end{tabular}



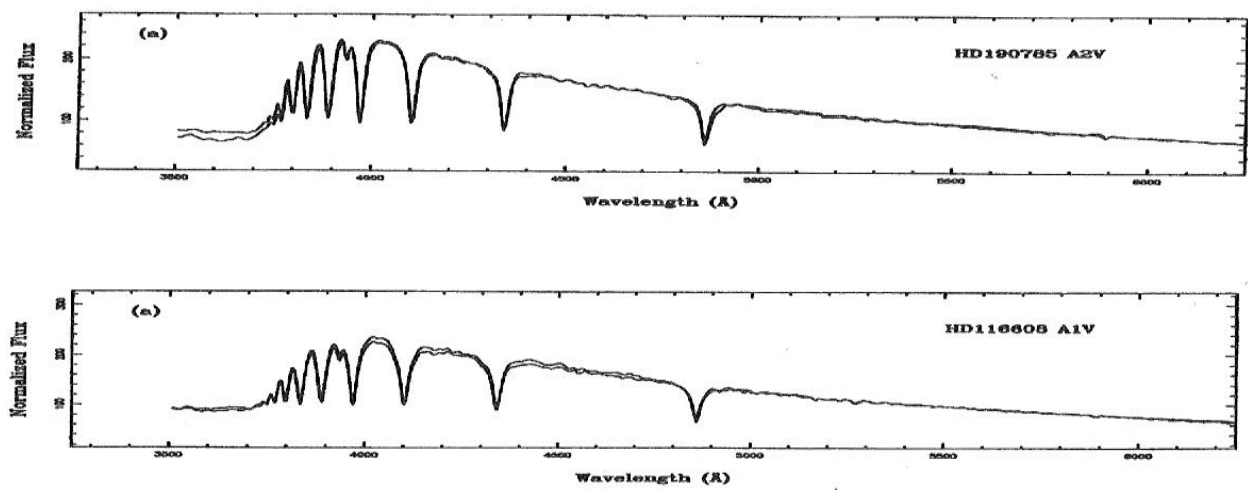

Fig 3 Plot of normalized flux versus the wavelength(Planck curve) for two different A-stars. The absorption hydrogen Balmer lines are clearly observed. The wavelength of the intensity maxima for both continuous and discrete emissions seems to be the same.

Table 4

$\begin{array}{lr}\text { Data from Table } 2 \text { used in Fig } \mathbf{4} \\ \text { A8 } & 1.75 \\ \text { A5-A7 } & 1.81 \\ \text { A1-A3 } & 1.84 \\ \text { B6V } & 1.88 \\ \text { B3-B4V } & 1.94\end{array}$

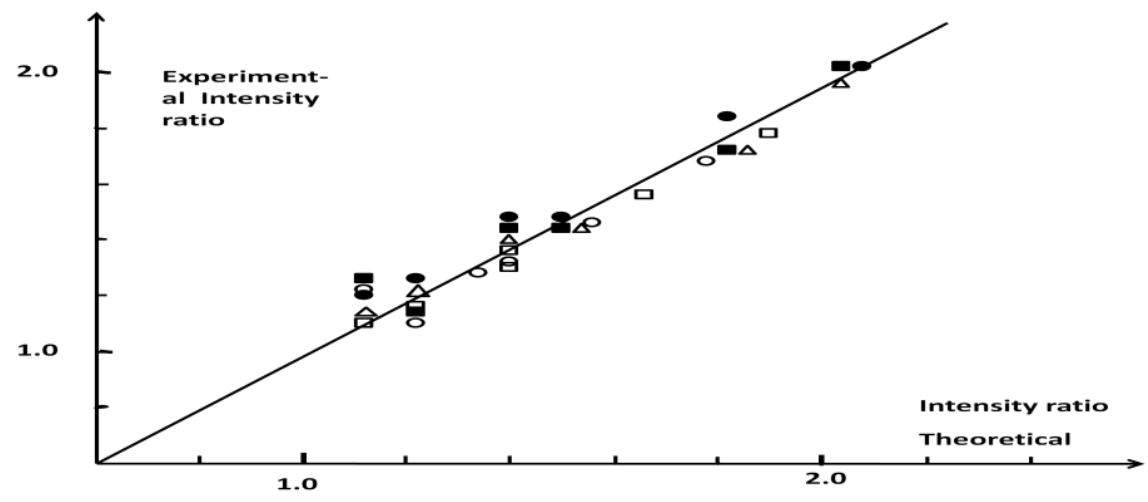

Fig 4 Spectral intensity ratios (experimental and theoretical) give very good correlation $(r=0.98)$ using the

Balmer lines from different spectral classes of stars using the new intensity formula.

Spectral classes used : A8=unfilled circles , A5-A7=unfilled squares, A1-A3=unfilled triangles, B6V= filled circles, B3-B4=filled squares.

\section{Determination of the effective temperature of a star}

Table (66 ) p.564 in Ref (10) were then used, where the effective temperatures were tabled from many main sequence stars from different spectral classes (A-K).These effective temperature values were then plotted versus the electron temperature values from corresponding spectral class from Table 2 in this paper. In this way effective temperature values have been obtained for 12 main sequence stars and are tabled in Table 5.

Good correlation $(+-85 \mathrm{~K})$ is here achieved between the values from this investigation and the literature values based on the Stefan-Boltzmann temperature law and can be seen in Table 5 and Fig 5, which show good correlation $(\mathrm{r}=0.99)$. 


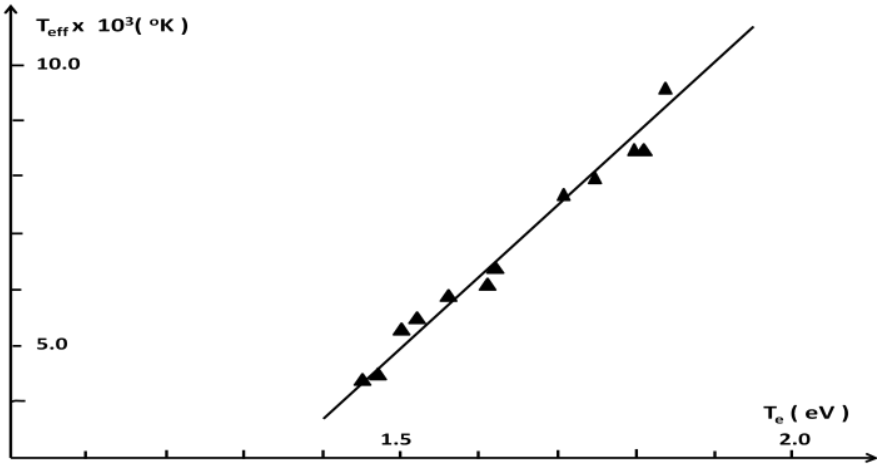

Fig 5 Effective temperature plotted versus electron temperature for a number of mainsequence stars. (correlation $\mathbf{r}=\mathbf{0 . 9 9})$

Table 5

Determination of $\mathbf{T}_{\text {effective }}$ of stars

\begin{tabular}{|c|c|c|c|}
\hline \multicolumn{2}{|c|}{ From the graph } & From the literature & Spectral group \\
\hline Vega & $9300^{\circ} \mathrm{K}$ & $9300^{\circ} \mathrm{K}$ & $\mathrm{A} 0$ \\
\hline Altair & 8100 & 8000 & A7 \\
\hline Procoyon A & 7500 & 7500 & F5 \\
\hline Sun & 5700 & 5740 & G2 \\
\hline Sirius A & 9500 & 9700 & A1 \\
\hline Aldebaran & 4200 & 4100 & K5 \\
\hline Pollux & 4700 & 4500 & K0 \\
\hline Capella B & 5200 & 4940 & G5-G0 \\
\hline Regulus & 9700 & 10300 & B7 \\
\hline Canopus & 7250 & 7350 & F0 \\
\hline Fomalhaut & 8700 & 8500 & A3 \\
\hline Sirius B & 8400 & 8200 & A5 \\
\hline
\end{tabular}

V. Determination of the density of a star by using Balmer lines.

According to equation 1 the $\mathrm{C}$-factor is a product of factors of number densities of atoms and electrons. By using the approximate formula of equation 1 we obtain :

$$
I=C \lambda^{-2} \exp \left(-(h v+J) / k T_{e}\right)
$$

By expressing $\mathrm{C}$ as a function of the other parameters in equation 5 and by taking the ratio between the density of a star compared to the sun, we obtain the following expression

$\mathbf{C}_{\text {star }} / \mathbf{1}=\left(\mathbf{I} \boldsymbol{Y}_{\text {star }} / \mathbf{I} \boldsymbol{Y}_{\text {sun }}\right)\left(\lambda_{\max \text { star }} / \lambda_{\max \text { sun }}\right)^{2} \exp \left(\left(\mathbf{h} \mathbf{V}_{\max \text { star }}+\mathbf{J}_{\mathrm{H}}\right) / \boldsymbol{\theta}_{\text {star }}-\left(\mathbf{h} \mathbf{v}_{\max \text { sun }}+\mathbf{J}_{\mathrm{H}}\right) /\right.$ $\left.\theta_{\text {sun }}\right)$

\section{( 6 )}

where $\mathrm{C}=1$ is the sun value and $\theta=\mathrm{kT}_{\mathrm{e}}$. The intensity ratio ( $\left.\mathrm{I} \gamma / \mathrm{I} \gamma_{\text {sun }}\right)$ here is the ratio between the $\gamma$-Balmer line from the star and the sun from the data of Ref (10). $\lambda_{\max }$ and the $h v_{\max }$ have also been taken from Ref (8) and the electron temperature values have been taken from Ref (9) for different spectral classes. $J_{H}$ is the ionization energy of hydrogen.

The results of 12 stars here, are shown in Fig 6 and Table 6 where $\rho / \rho_{0}$-values been calculated for 12 different main sequence stars. In Fig 6 a straight line is achieved following in the near of the Schwarzschild line Ref 10 (p.555).

Table 6

Determination of density ratio of 12 stars relative to the sun

$\begin{array}{lcc}\text { Star } & \rho / \rho_{\text {sun }}(\text { new method }) & \text { spectral class } \\ \text { Aldebaran } & 1.26 & \text { K5 } \\ \text { Pollux } & 0.95 & \text { K0 } \\ \text { Capella B } & 0.91 & \text { G5 } \\ \text { Sun } & 1.0 & \text { G2 } \\ \text { Procyon A } & 0.66 & \text { F5 } \\ \text { Canopus } & 0.55 & \text { F0 }\end{array}$




$\begin{array}{lcc}\text { Altair } & 0.45 & \text { A7 } \\ \text { Sirius B } & 0.37 & \text { A5 } \\ \text { Formalhaut } & 0.42 & \text { A3 } \\ \text { Sirius A } & 0.42 & \text { A1 } \\ \text { Vega } & 0.39 & \text { A0 } \\ \text { Regulus } & 0.28 & \text { B7 } \\ \eta \text { Ori } & 0.14 & \text { B1 }\end{array}$

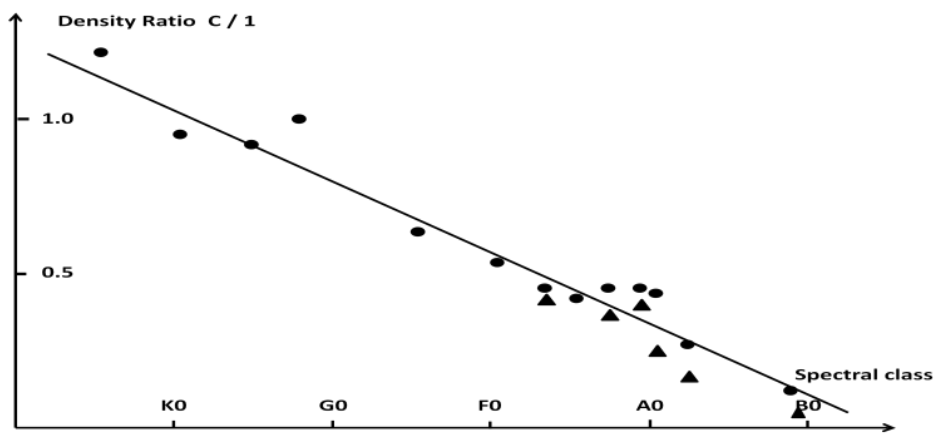

Fig 6 Density determination of stars relative to the sun at different spectral classes. Filled circles = new method , Filled triangles $=$ Schwarzschild limit

VI. Discussion

This method of analysis has shown to be a simple method of verifying the new intensity formula by using atomic and stellar data. By using this method together with the new intensity formula it has been possible to determine the mean electron temperature in different laboratory plasmas and in the optical layers of a star without knowing so much about the chemical composition of the star. These mean electron temperature values fit well with other methods from the literature. The method also gives an organizing method for stars similar to the established HR-diagram. The $\mathrm{J}_{\text {meanvalue }}$ has shown to be a kind of "signum" for every star. Fig 2 have shown to be a valuable and simple method of organizing and classifying the stars without knowing so many other details about the stars.

It has also been possible to determine the effective temperature of a number of stars from different spectral classes on the main sequence. The results gave good agreement with the established temperature method by Stefan-Boltzmann.

The Balmer spectral absorption lines seem to follow the new intensity formula too, which is clearly seen in Figs 3 and 4.This is clearly seen by the correlation coefficient ( 0.98$)$. This means that discrete emissions in the star do follow the new intensity formula but are heavily absorbed in the star. Therefore, the light coming from the star is mostly continuous radiation following Planck radiation law.

It has also been possible to determine the density of a number of stars compared to the sun from different spectral classes on the main sequence. These values are in accordance with the Schwarzschild limit. The graph in Fig 6 shows a nice linear relationship.

\section{Acknowledgement:}

I would like to express my gratitude to my collegue and friend Dr. Sten Yngström at the Swedish Institute of Space Physics for valuable and interesting discussion about this work.

\section{References:}

[1] S. Yngström, Internat. Journal of Theoret. Physics, 33, No 7, 1479 (1994)

[2] B. Thelin and S. Yngström, S, Spectrochim.Acta 41B , 403 (1986)

[3] S. Yngström and B. Thelin, Appl. Spectrosc. 44, 1566 (1990)

[4] W.F. Meggers, C.H. Corliss, and F. Scribner, Tables of Spectral Line Intensities, National Bureau of Standards ,Monograph 32 Part 1,Washington D.C. (1961)

[5] B. Thelin, Eurasian J. Anal. Chem. 4 (3) p.226, (2009)

[6] B. Thelin, African Phys Review, 4 (2010), 121

[7] M.Kaminsky, Atomic \& Ionic Impact Phenomena on Metal Surfaces, Springer Verlag, Berlin, Heidelberg,New York , 1965

[8] D.R. Silva and M.E. Cornell, Astrophysical Journal Suppliment Series, 81, No2, Aug. (1992)

[9] B. Thelin, Fizika B, 19, 4 (2010) 329

[10] K.R. Lang, Astrophysical Formula, Springer Verlag, Berlin, Heidelberg, New York, (1974) 